\title{
Subfactors and Conformal Field Theory
}

\author{
DAVID E. EVANS \\ University of Wales, Swansea, SA2 8PP, WALES \\ and \\ YASUYUKI KAWAHIGASHI \\ University of Tokyo, Tokyo, 113, JAPAN
}

(Received: November 6, 1992)

\begin{abstract}
We discuss relations between the combinatorial structure of subfactors, solvable lattice models, (rational) conformal field theory, and topological quantum field theory.

Key words: conformal field theory, modular automorphism group, modular invariant, orbifold construction, statistical mechanics, subfactors, topological invariant
\end{abstract}

\section{Introduction}

Since the breakthrough by V. F. R. Jones [48, 49], the theory of subfactors has had unexpectedly deep connections with topology and mathematical physics. The combinatorial structure of subfactors plays a key role, and Ocneanu's paragroup theory [64] gives the most powerful machinery for understanding this at present. Ocneanu, however, has not published details of his theory, and a lack of understanding of the paragroup theory prevented detailed study of the relationship between the paragroup theory and the topology and physics - beyond noticing apparent similarities in the various fields. Recently, full details and several applications of the paragroup theory have appeared $[11,32,33,34,42,44,45,52,53,54,66,67,83]$ and our aim here is to give a full account of the recent interactions.

A paragroup is a (discrete) quantization of a (finite) group, and it appeared as a combinatorial characterization of higher relative commutants of finite index and finite depth subfactors of the approximately finite dimensional (AFD) factor of type $\mathrm{II}_{1}$. (See [38] for the basics of subfactor theory and [71] for analytic aspects of the classification of subfactors.) It has been realized that the combinatorial structure of paragroups has considerable similarity with several other structures in mathematics and physics. The following table shows correspondences between various notions in paragroup theory, exactly solvable lattice model theory, quantum $6 j$-symbols, rational conformal field theory and topological quantum field theory. Further details on these will be presented in later sections. Some correspondences are more 
exact than others — some merely express similarity.

\begin{tabular}{|l|l|l|l|l|l|}
\hline $\begin{array}{l}\text { Paragroups } \\
\text { (path } \\
\text { algebras) }\end{array}$ & $\begin{array}{l}\text { Paragroups } \\
\text { (bimodules) }\end{array}$ & $\begin{array}{l}\text { Lattice } \\
\text { models }\end{array}$ & $\begin{array}{l}\text { Quantum } \\
6 j \text { - } \\
\text { symbols }\end{array}$ & RCFT & TQFT \\
\hline \hline \multicolumn{7}{|c|}{ connection } & $\begin{array}{l}\text { Boltzmann } \\
\text { weight }\end{array}$ & $6 j$-symbol & $\begin{array}{l}\text { braiding } \\
\text { matrix }\end{array}$ & $\begin{array}{l}\text { local } \\
\text { data for } \\
\text { tetrahedron }\end{array}$ \\
\hline \multicolumn{5}{|c|}{ unitarity } & $\begin{array}{l}\text { invariance } \\
\text { under } \\
\text { moves II, } \\
\text { III }\end{array}$ \\
\hline $\begin{array}{l}\text { commuting } \\
\text { square }\end{array}$ & $\begin{array}{l}\text { Frobenius } \\
\text { reciprocity }\end{array}$ & $\begin{array}{l}\text { crossing } \\
\text { symmetry }\end{array}$ & $\begin{array}{l}S_{4^{-}} \\
\text {symmetry }\end{array}$ & $\begin{array}{l}\text { tetrahedral } \\
\text { symmetry }\end{array}$ \\
\hline flatness & associativity & $\begin{array}{l}\text { Yang- } \\
\text { Baxter } \\
\text { equation }\end{array}$ & $\begin{array}{l}\text { pentagon } \\
\text { relation }\end{array}$ & $\begin{array}{l}\text { braiding- } \\
\text { fusion } \\
\text { relation }\end{array}$ & $\begin{array}{l}\text { invariance } \\
\text { under } \\
\text { move I }\end{array}$ \\
\hline
\end{tabular}

See $[52,53,65,66,74]$ for the path algebra approach to paragroups, and $[33,66,67,84]$ for the bimodule approach to paragroups.

This work was partially supported by financial support from the British Council, Miller Research Fellowship, MSRI (Berkeley), SERC, and University College of Swansea. We thank Professors D. Bisch, M. Choda, U. Haagerup, M. Izumi, V. F. R. Jones, A. Kato, T. Kohno, H. Kosaki, T. Miwa, R. Nest, A. Ocneanu, M. Okado, S. Okamoto, S. Popa, N. Yu. Reshetikhin, Ph. Roche, M. Rosso, M. Wakui, Y. Watatani, H. Wenzl, F. Xu, S. Yamagami, and J.-B. Zuber for several useful comments and or conversations during this work.

\section{Statistical Mechanics and Subfactors}

We enter this maze of interactions involving operator algebras, conformal quantum field theory, and topological quantum field theory, with certain statistical mechanical models on a two dimensional infinite oriented lattice $L$ (which for our purposes here will be $\mathbf{Z}^{2}$ ). The configuration space of the classical model will be (compatible) distributions of the edges of some oriented graph $\Gamma$ (here taken to be finite) on the edges of $L$. If there is at most one edge between any pair of vertices of $\Gamma$, and if there is an edge from $\alpha$ to $\beta$ then there is another edge from $\beta$ to $\alpha$, then this will be the same as distributing the vertices of $\Gamma$ over $L$ so that labels of two vertices in $L$ are joined in $\Gamma$ if the original vertices are joined in $L$. For the Dynkin diagram $A_{3}$ with vertices labeled as 


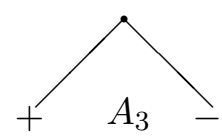

and square lattice $L$ (with downward orientation)

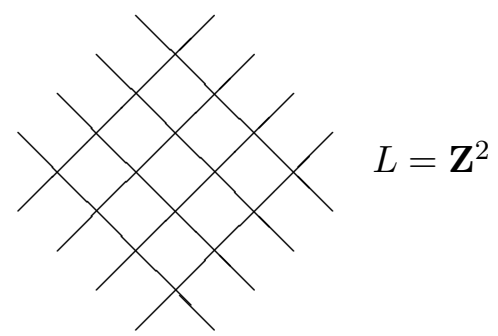

one obtains two copies of the configuration space of the Ising model by placing the frozen spin - on the even or odd sublattices of $L$.

$$
\begin{aligned}
& \pm \\
& \pm \\
& \pm
\end{aligned}
$$

To complete the data for the model, we assign a complex number $W(\alpha, \beta, \gamma, \delta)$, the Boltzmann weight to each allowed configuration:

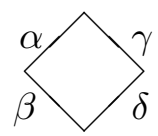

For the $A_{3}$ graph, we assign the appropriate Ising weights to the eight possible configurations:

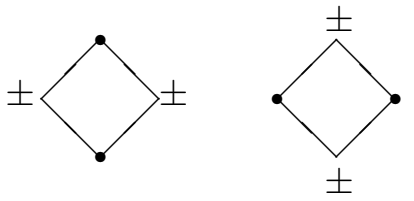

The Andrews-Baxter-Forrester models are associated with the Dynkin diagrams $A_{n}[2]$ :

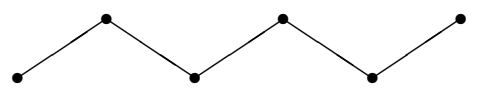


Thinking of this as an oriented graph with basic transitions we can generalize to the Weyl alcove $A^{(n)}$ of the level $k$-integrable representations of the Kac-Moody algebra $\mathcal{A}_{N-1}^{(1)}=S \widehat{U(N)}$. For $S U(3)$ instead of $S U(2)$, we replace

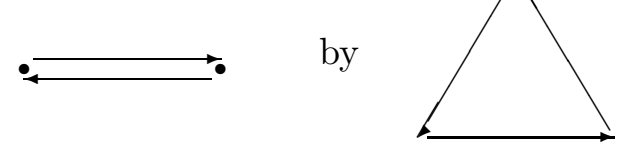

i.e., transitions generated by 3 vectors (instead of 2) which add to zero. Let

$$
P_{++}^{(n)}=\left\{\lambda=\sum_{i=1}^{N-1} \lambda_{i} \Lambda_{i} ; \lambda_{i} \geq 1, \sum_{i=1}^{N-1} \lambda_{i} \leq n-1\right\},
$$

where the $\Lambda_{i}$ 's are the $N-1$ weights of the fundamental representations and $n=k+N$. The vertices of the graph $\mathcal{A}^{(n)}$ are the elements of $P_{++}^{(n)}$ and its oriented edges are given by $N$ vectors $e_{i}$ defined by

$$
\begin{aligned}
e_{1} & =\Lambda_{1}, \\
e_{i} & =\Lambda_{i}-\Lambda_{i-1}, \quad i=1,2, \ldots, N-1, \\
e_{N} & =-\Lambda_{N-1} .
\end{aligned}
$$

These models were introduced and studied by Date, Jimbo, Miwa and Okado [21].

The notion of an orbifold of such a model, dividing out by a symmetry group $G$ on $\Gamma$ which preserves the Boltzmann weights was introduced by $[36,37,22]$. The model based on $\Gamma$ is replaced by one built on an orbifold graph, denoted by $\Gamma / G$; i.e., we consider a model on the orbifold graph $\Gamma / G$ equipped with some Boltzmann weights. Fendley and Ginsparg considered the $\mathbf{Z}_{2}$-orbifold of the $A$-series associated to $S U(2)$ and Di Francesco and Zuber for the $\mathbf{Z}_{3}$-orbifolds of the corresponding $S U(3)$ orbifolds.
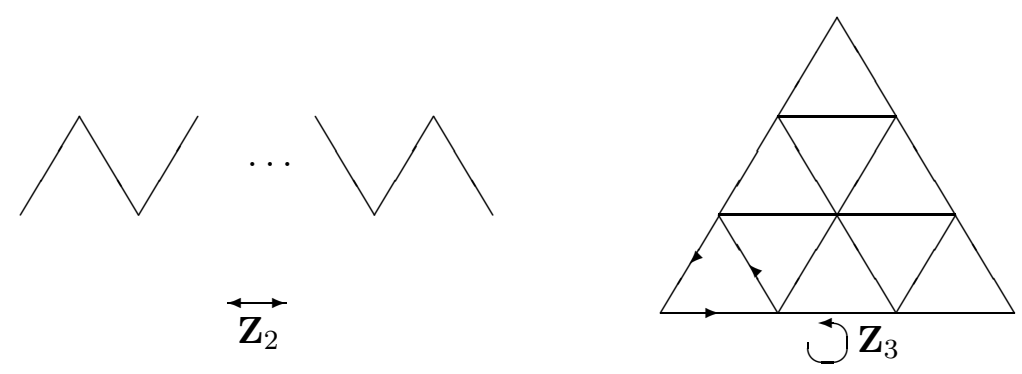
In particular, they expressed the Boltzmann weights for the original $\mathcal{A}^{(n)}$ _ model (for $N=2,3$ respectively) as

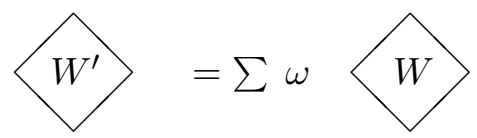

with complex coefficients $\omega^{N}=1$, and could show by direct computation that the Yang-Baxter equation for the orbifold graph was inherited from the corresponding equation for the original graph. In this framework, the Yang-Baxter equation is expressed as equality of the partition functions

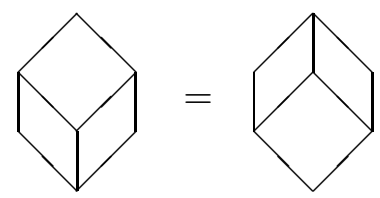

where both figures have the same configurations (edges of $\Gamma$ ) along the boundary and the sum (the product of 3 Boltzmann weights) over internal edges.

The orbifold procedure is best understood in the path algebras associated to graphs. The transfer matrix method transforms the two dimensional classical model to a one-dimensional quantum system $[70,60,35,3,13,58$, 30]. The classical observables are $C(P)$, the commutative $C^{*}$-algebra of all continuous functions on the compact Hausdorff space of configurations, and at each inverse temperature $\beta$ we may be interested in the simplex $K_{\beta}$ of equilibrium states. The transfer matrix formalism transfers this setting to that of a one dimensional quantum model represented by a non-commutative $C^{*}$-algebra $A$, a path algebra on the graph $\Gamma$ or the algebra generated by the local transfer matrices. For each inverse temperature $\beta$, one looks for a map from equilibrium states in $K_{\beta}$ to linear functionals on $A$ and a map $F \mapsto F_{\beta}$ from (local) classical observables in $C(P)$ to the quantum algebra $A$ such that one recover the classical expectation or correlation functions from a knowledge of the quantum ones alone: $\mu(F)=\varphi_{\mu}\left(F_{\beta}\right)$.

The non-commutative path algebra $A(\Gamma)$ associated to the graph $\Gamma$ is defined as follows. First take the space of all one-sided paths in $\Gamma$ (beginning in some specified subset of vertices of $\Gamma$ ). Drawing the paths vertically, beginning with the initial vertices at level zero, we go down one level at a time, using the graph $\Gamma$, so that at each level we have a family of vertices. Consider the finite dimensional algebra $A[m, n]$ between the two levels $m$ and $n$, generated by matrix units or partial isometries $|\xi\rangle\langle\eta| \equiv(\xi, \eta)$, where $\xi, \eta$ are paths from level $m$ to $n$, with $(\xi, \eta)=0$ unless $\xi, \eta$ have the same initial and same terminal vertices. Let $A(\Gamma)=\lim A[m, n]$ be the completion, i.e., the 
$C^{*}$-algebra generated by such pairs of paths $(\xi, \eta)$, with $A[m, n]$ embedded into $A\left[m^{\prime}, n^{\prime}\right],\left(m^{\prime} \leq m \leq n \leq n^{\prime}\right)$ by

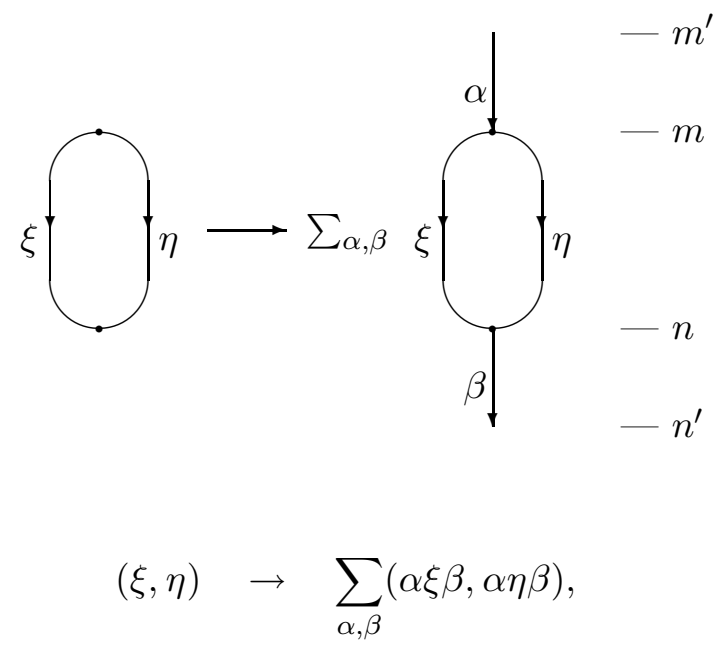

where the summation is over paths $\alpha$ from level $m^{\prime}$ to level $m, \beta$ from level $n$ to $n^{\prime}$.

Writing

$$
W=\sum \sum_{\beta}^{\alpha}(\alpha \beta, \gamma \delta)
$$

we see that being given a (translation invariant) Boltzmann weight is the same as begin given an element $W$ in the path algebra $A[n-1, n+1]$, for some $n$. Then for any $n$, letting $W$ be the corresponding element of the path algebra, the Yang-Baxter equation corresponds to

$$
W_{n} W_{n+1} W_{n}=W_{n+1} W_{n} W_{n+1} .
$$

In general, the $\left\{W_{n} ; n \in \mathbf{N}\right\}$ will not generate the path algebra, but they will in the case of the $\mathcal{A}^{(n)}$-models of [21], when $W_{n}$ gives a representation of the Hecke algebra of type $A$. If the group $G$ acts on $\Gamma$, then (under reasonable hypotheses) the fixed point algebra $A(\Gamma)^{G}$ is isomorphic to $A(\Gamma / G)$, the path algebra of the orbifold graph; with this isomorphisms explicitly leading to (2), as a Boltzmann weight invariant under the group action gives an invariant element of the path algebra $A(\Gamma)$. This gives a natural meaning for the construction of the orbifold graph. The fixed points of the graph $\mathcal{A}^{(n)}$ are replaced by $N$ vertices representing the orbit and, the $N$ vertices which are moves cyclically onto each other are replaced by singleton as in the following examples: 


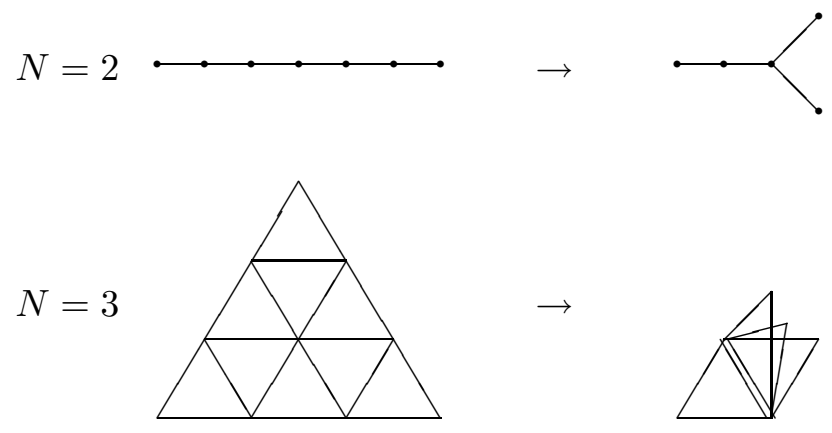

These models were first introduced by Pasquier [69] in the case of $N=2$ by an ad hoc method of finding Boltzmann weights for the $D$-series which satisfies the Yang-Baxter equation, and later by $[36,37]$ for the case $N=2$, $N=3$, who used (1) to write down the Boltzmann weights and verified by hand that they satisfied the Yang-Baxter equation. Di Francesco and Zuber predicted similar models for general $N$ would follow as a consequence of identities between theta functions. The methods of [32] shows that the best way to understand these models is via the path algebra picture outlined here. (Pasquier also used ad hoc methods to obtain a model at criticality for $E_{6}$; an embedding of $A_{11}$ in $E_{6}$ via the Temperley-Lieb algebra.) This is a discrete version of orbifold theory in the structure theory of $C^{*}$-algebras, where the existence of fixed points in the symmetry group of some underlying structure tends to new phenomena. For example, consider the $\mathbf{Z}_{2}$-action of flipping the circle; $z \mapsto \bar{z}$. The crossed product of a pair of distinct points $\{z, \bar{z}\}$ moved by a symmetry $\mathbf{Z}_{2}$ is $C\{z, \bar{z}\} \times \mathbf{Z}_{2}=M_{2}$, the $2 \times 2$-complex matrices generated by the diagonal $\mathbf{C}^{2}$, and the $\mathbf{Z}_{2}$-flip which interchanges the components. The crossed product of a singleton $\{z\}$ by $\mathbf{Z}_{2}$-action is $\mathbf{C}^{2}$ $\left(=C\left(\hat{\mathbf{Z}}_{2}\right)\right)$. Gluing this together, the crossed product $C(\mathbf{T}) \times \mathbf{Z}_{2}$ is the $M_{2^{-}}$ valued functions on the interval (or a semicircle) diagonal at the endpoints, represented geometrically as

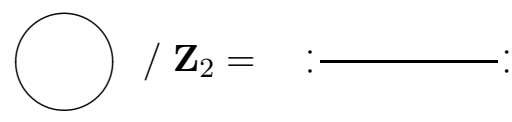

This example is the continuous analogue of (3) and is at the heart of recent progress in the structure theory of amenable $C^{*}$-algebras, where for example, one can show the existence of compact group actions on AF algebras whose fixed point algebras are not $\mathrm{AF}[5,59,6,7,8]$. The analogous $\mathbf{Z}_{2}$-action on the non-commutative two torus $A_{\theta}$ leads to an AF algebra $A_{\theta} \times \mathbf{Z}_{2}[9,10]$ (so that the dual action has again non-AF fixed point algebra, essentially $A_{\theta}$ ); 
the fixed point algebra $A_{\theta}^{\mathbf{Z}_{2}}$ is also AF, and this may help in understanding the Cantor spectra of discrete Schrödinger operators with almost periodic potentials.

Returning to our central framework, note that the graph $A_{3}$ is self-dual

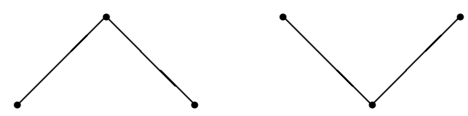

In this case, the process taking the orbifold model, is a non-trivial identification of the Ising model with itself; indeed it is the high temperature-low temperature duality

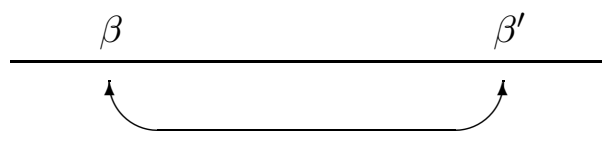

of Kramers and Wannier [19, 35]. Note that again the existence of a fixed point under this transformation is what leads to the interesting phenomena; it is the critical temperature of Onsagar $\beta_{c}$.

We now use the path formulation of AF algebras [28, 29, 64, 65, 75] to construct subfactors, with the main interest centered on those constructed from the solvable lattice models. Consider the following

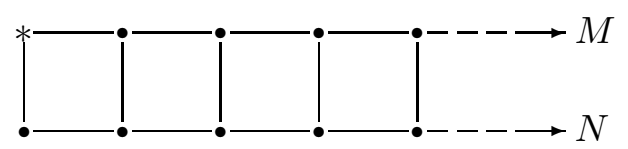

Here each line represents a graph, and each node represents a sequence of vertices say perpendicular to the plane of the paper, where at the top left hand corner we have one distinguished vertex * Taking the Bratteli diagrams

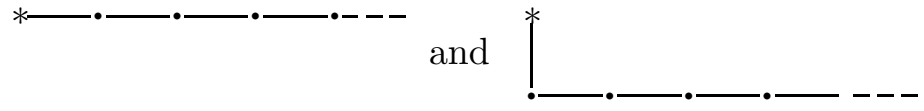

we obtain two AF algebras, completing with respect to some traces we obtain von Neumann algebras $N$ and $M$; the vertical graphs will allow us to embed $N$ in $M$ - for the time being we will not be precise about this. We want to extend this picture to obtain a double complex and a sequence of factors $N \subset M \subset M_{1} \subset \cdots$ : 


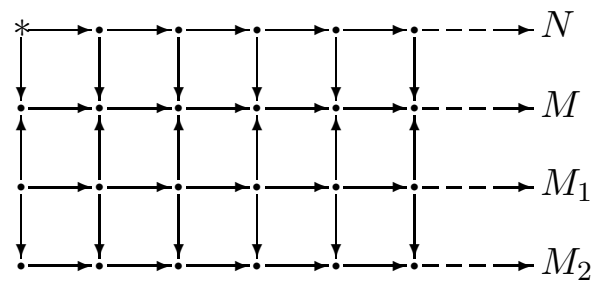

We do not wish to generate a random sequence of factors, but the Jones tower associated to the first inclusion $N \subset M$. The Jones basic extension from $N \subset M$ to $M \subset M_{1}$ can be thought of as a reflection of $N$ through $M$; this can be achieved by reflecting the vertical graphs as indicated by alternating the orientations. This using the graphs $\mathcal{A}^{(n)}$, where the vertices are $N$-colorable, we obtain the following picture of colors [32]:

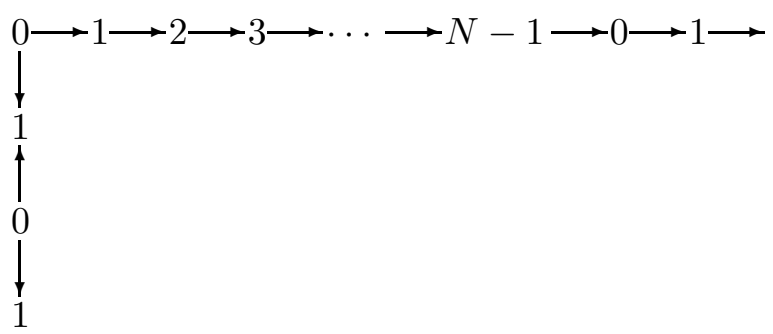

The Jones tower of relative commutants $N^{\prime} \cap N \subset N^{\prime} \cap M \subset N^{\prime} \cap M_{1} \subset$ $N^{\prime} \cap M_{2} \cdots$ is an inclusion of finite dimensional $C^{*}$-algebras in the case of finite index; its Bratteli diagram being the iteration of a single graph called the principal graph $\Gamma$ (which may bear no relation in general to the graphs used in (4) to construct the subfactor). The inclusion $N \subset M$ is said to be of finite depth when the principal graph is finite, in which case the norm of its incidence matrix is the (square root) of the Jones index of the subfactor. Our task is to identify the principal graph in certain examples.

To compute the relative commutant $N^{\prime} \cap M_{k}$

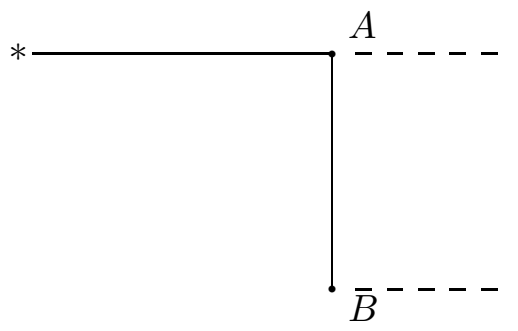

first consider at the finite dimension level the commutant $A^{\prime} \cap B$. Straightening this out, 


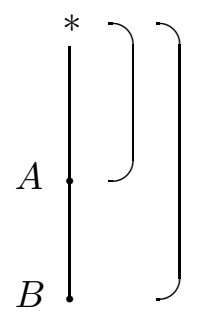

we need the commutant of a path algebra $A[0, n]$ in $A[0, m]$ which is easily seen to be $A[n, m]$, the vertical path algebra

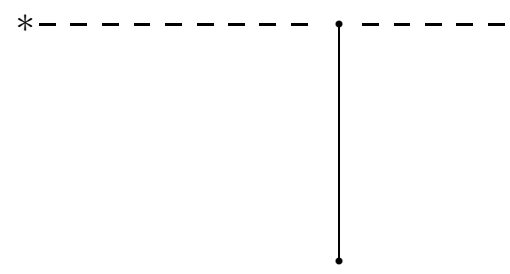

Since we need the relative commutant at the infinite stage, it is reasonable to expect (and it is the case when the four basic graphs in (4) are finite) that what we should take is that part of the vertical algebra which is transformed

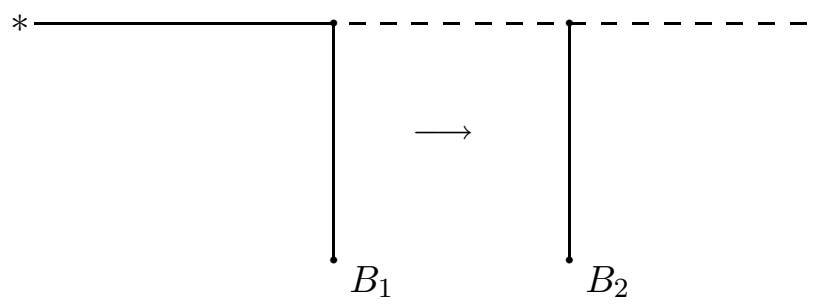

to another vertical path algebra when we embed $B_{1}$ in $B_{2}$. This is the notion of parallel transport of Ocneanu [64, 66]; the principal graph is general being the flat part (of a given complex (5)). The simplest case to describe is when everything vertical transforms in a parallel fashion to a vertical algebra; in which case the principal graph can be identified with the graph appearing in the left most vertical part of the complex (5).

So far, we have not been specific about the vertical inclusions. If we take trivial diagonal embeddings

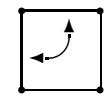

then we would be in the worst possible situation where something vertical becomes horizontal. We need to make a non-diagonal identification, and 
this is where the Boltzmann weights become useful. They provide us, with suitable specialization of the spectral parameter, with a unitary to identify the path algebra $\_$with $\vec{\downarrow}$ and when normalized, they give rise to a unitary to identify paths $\longleftrightarrow$ with $\vec{\jmath}$. This is a connection in the sense of Ocneanu $[64,65,66]$; the unitarity condition corresponds to the first inversion relation in the solvable lattice model theory, the biunitarity (unitarity of $\vec{L}$ under renormalization) or commuting square condition corresponds to the second inversion relation. It is the Yang-Baxter equation which is useful in helping to compute the flat part of a graph. By symmetry, flatness is the same as vertical parallel transport of the horizontal path algebras. Thus taking a face operator in the horizontal path algebra we have [73]:
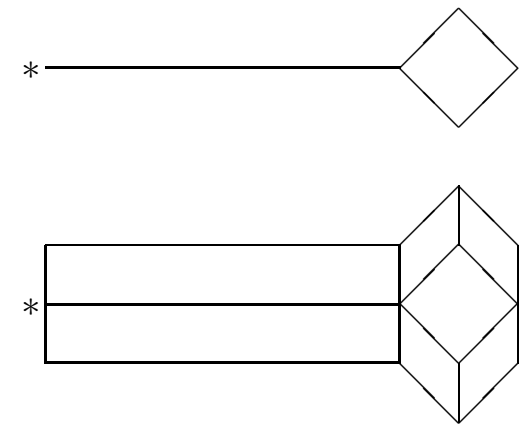

using the connection

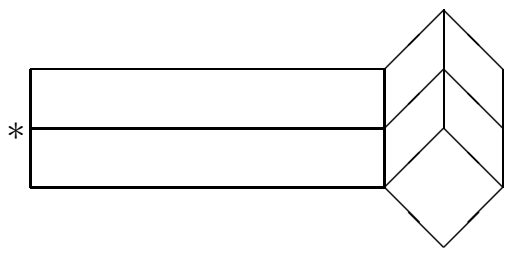

by Yang-Baxter equation

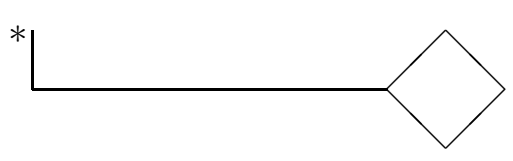

by unitarity

Thus if face operators generate the (first) horizontal algebra in (2), then we certainly have flatness. This is the case for the $\mathcal{A}^{(n)}$ models with connection given by the Boltzmann weights [46] so that the principal graphs of these subfactors (first constructed by Wenzl of [80] who computed their index) are their 0-1 parts [32] (c.f. (3)). Similarly embedding $A_{11}$ in $E_{6}$ (since a Temperley-Lieb algebra can be embedded in the path algebra of a bipartite graph [38]), the principal graph is the intertwining graph (computed in [69, 31]) and so we recover the result of Okamoto [68]. Note that here we are using 
the intertwining Yang-Baxter equation between the (critical) Boltzmann weights on $A_{11}$ and $E_{6}$ (see [22]).

\section{Modular Invariants and Subfactors}

The $A-D-E$ classification of (level $k$ ) $S U(2)$ Wess-Zumino-Witten models is as follows. The $S U(2)_{k}$ Kac-Moody algebras has $k+1$ integrable representations $\left[\varphi_{j}\right], 2 j=0, \ldots, k$, with $S U(2)$ isospin $j$ and affine character $\chi_{\lambda}(\tau)=\operatorname{Tr}_{\mathcal{H}_{j}} e^{2 \pi \tau i\left(L_{0}-c / 24\right)}, \lambda=2 j+1$. A one loop partition function on the torus should be expressed as $Z\left(\tau, \tau^{\prime}\right)=\sum N_{\lambda, \bar{\lambda}} \chi_{\lambda}(\tau) \overline{\chi_{\bar{\lambda}}}\left(\tau^{\prime}\right)$. (a continuum version of the transfer matrix formalism outlined above). The classification problems to find all such partition functions of the above form, where $N_{\lambda, \lambda^{\prime}}$ are non-negative integers (arising from a decomposition of the underlying Hilbert space as $\oplus N_{\lambda, \bar{\lambda}} \mathcal{H}_{\lambda} \otimes \mathcal{H}_{\bar{\lambda}}$ ), and where $Z$ is modular invariant (due to invariance under reparametrizations of the torus). Such modular invariant sesqui-linear forms were shown $[12,50]$ to be of type $A-D-E$.

The identification of a modular invariant with a graph is through noting that the diagonal part (writing $Z=\sum_{\lambda \in \mathcal{E}}\left|\chi_{\lambda}\right|^{2}+$ remainder) of a modular invariant is described by a set $(\mathcal{E})$ of exponents of an $A-D-E$ Dynkin diagram. Thus for $k=16$, we have three invariants corresponding to $A_{17}, D_{10}, E_{7}$ as follows:

\begin{tabular}{|l|l|l|}
\hline modular invariant & exponents & graph \\
\hline \hline$\left|\chi_{1}\right|^{2}+\left|\chi_{2}\right|^{2}+\cdots+\left|\chi_{17}\right|^{2}$ & $1,2,3, \ldots, 17$ & $A_{17}$ \\
\hline$\left|\chi_{1}+\chi_{17}\right|^{2}+\left|\chi_{3}+\chi_{15}\right|^{2}+\mid \chi_{5}+$ & $1,3,5,7,9,11,13,15,17$ & $D_{10}$ \\
$\left.\chi_{13}\right|^{2}+\left|\chi_{7}+\chi_{11}\right|^{2}+2\left|\chi_{9}\right|^{2}$ & & \\
\hline$\left|\chi_{1}+\chi_{17}\right|^{2}+\left|\chi_{5}+\chi_{13}\right|^{2}+\mid \chi_{7}+$ & $1,5,7,9,11,13,17$ & $E_{7}$ \\
$\left.\chi_{11}\right|^{2}+\left|\chi_{9}\right|^{2}+\left(\chi_{3}+\chi_{15}\right) \chi_{9}^{*}+$ & & \\
$\chi_{9}\left(\chi_{3}+\chi_{15}\right)^{*}$ & & \\
\hline
\end{tabular}

In the subfactor theory, only $A-D-E$ Dynkin diagrams, with $D_{\text {even }}$ and $E_{\text {even }}$ appear as principal graphs of subfactors with index less than four. In the rational conformal field theory of $S U(2)$ Kac-Moody WZW models, described by $A-D-E$ Dynkin diagrams, there is a degeneracy so that only $D_{\text {even }}$ and $E_{\text {even }}$ need be counted. For example in the case of $k=16$, the modular invariant for $E_{7}$ reduces to that of $D_{10}$ under the simple interchange of blocks $\chi_{9}$ and $\chi_{3}+\chi_{15}$. Note that there are two kinds of modular invariants here

$$
\begin{array}{ll}
\sum\left|\chi_{i}\right|^{2} & \text { type I } \\
\sum \chi_{i} \bar{\chi}_{\sigma(i)} & \text { type II }
\end{array}
$$

where $\sigma$ is a permutation of the extended fusion rules. In this case, the flip on the extended blocks identifies the two modular invariants for $D_{10}$ and $E_{7}$, 
and led Zuber to conjecture that the subfactor built from the $E_{7}$ connection

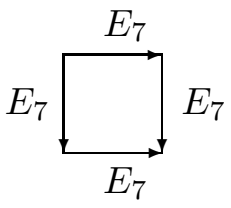

is the $D_{10}$ subfactor (i.e., the flat part of $E_{7}$ is $D_{10}$ ). This conjecture was verified in [34]; indeed using a commuting square or connection

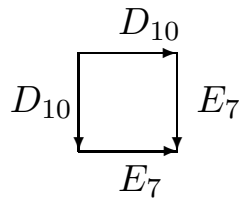

where the two $D_{10}$ 's are glued together by a non-trivial identification corresponding exactly to the interchange blocks $\chi_{9} \leftrightarrow \chi_{3}+\chi_{15}$.

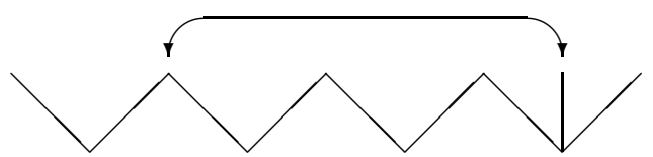

This is related to an observation in [24].

Note that the flat parts of the other Dynkin diagrams had been known; $A_{n}, D_{2 n}, E_{6}, E_{8}$ are flat and $D_{2 n+1}, E_{7}$ are non-flat as announced by Ocneanu [64] and proved by $[4,43,44,52,76]$. It is easy to see that the flat part of $D_{2 n+1}$ is $A_{4 n-1}$ and again corresponds to a degeneracy of a type II modular invariant for $D_{2 n+1}$ to a type I invariant for $A_{4 n-1}$. See also [42] for an approach to flatness based on ideas from quantum groups.

\section{Rational Conformal Field Theory (RCFT) and Subfactors}

The combinatorial approach to rational conformal field theory such as $[63$, 82] has much in common with paragroup theory, and was explicitly worked out in [11] for the first time. We will explain this work [11] briefly and then show how $\mathrm{F} . \mathrm{Xu}[83]$ combined this method and the orbifold construction of $[32,52]$. The operator algebraic meaning of this construction will be clarified in Section 6.

Moore and Seiberg [63] showed that the braiding and fusion matrices and the modular matrix satisfying certain axioms contain the duality data of a conformal field theory. If we start from a connected, simply connected, 
compact and simple Lie group $G$ and an integer $k$ called a level, one gets such combinatorial data and can compute an expectation value of knotted graphs as in [82]. In [11], de Boer and Goeree showed that this kind of combinatorial data produce a paragroup from an RCFT and a choice of a primary field. The biunitary connection is directly obtained from entries of the braiding matrices, and the only non-trivial axiom we have to verify is flatness. Their observation is that if we have a partition function diagram with $*$ at all the four corners, the computation of the value is reduced to that of the expectation value of the following knotted diagram.

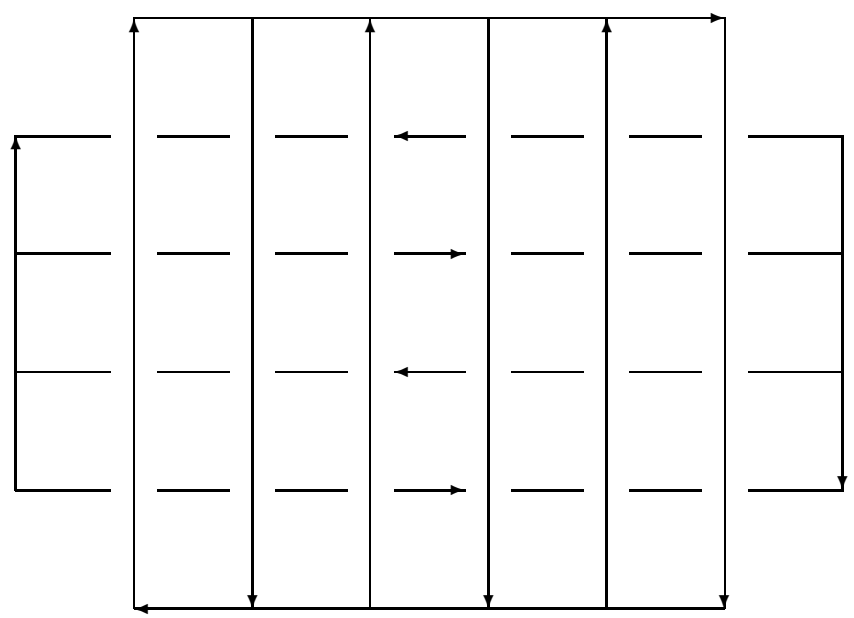

This diagram can be split into two pieces because the vertical arrows are over the horizontal arrows at all the crossings. Then it is very easy to compute the expectation value and we get flatness of the connection.

Next we explain the orbifold construction of subfactors from this viewpoint. The orbifold construction was first used in subfactor setting in [52] to prove classification of subfactors corresponding to the Dynkin diagram $D_{n}$ which was announced by Ocneanu without a proof in [64], and established as a general method in [32]. The main idea is that if a paragroup has a symmetry of finite order, we can produce a candidate for a new paragroup which is the quotient of the original paragroup by the symmetry. This is interesting especially when the symmetry moves the distinguished vertex $*$ of the paragroup. In such a case, the symmetry cannot act on the higher relative commutants naturally, but we realize the subfactor in non-canonical way so that the symmetry can act. That is, the path algebra starts not just from $*$ but from all the images of $*$ under the symmetry. Then we can take fixed point algebras of the double sequence of the path algebras and get a natural biunitary connection on the double sequence of the fixed point algebras, then the only remaining problem is to verify flatness. The paragroups 
corresponding to the Dynkin diagrams $A_{2 n-3}$ naturally have $\mathbf{Z}_{2}$-symmetries, and the fixed point algebras under the $\mathbf{Z}_{2}$-action on the double sequence of the path algebras starting from the two endpoints of $A_{2 n-3}$ give the connection on $D_{n}$. Then the flatness problem of $D_{n}$ is reduced to the following identity of the partition functions as in [52].

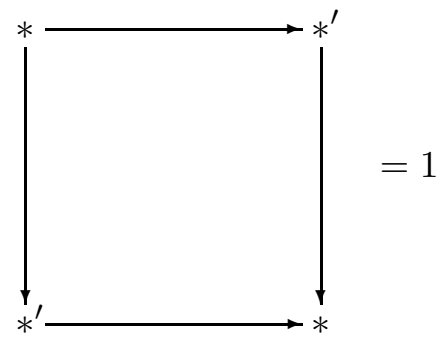

where $*^{\prime}$ is the other endpoint than $*$, and the size of the diagram is $(2 n-$ $4) \times(2 n-4)$. Then direct computation based on induction as in [52] shows that the value of the left hand side is $(-1)^{n}$, and this proves that the Dynkin diagrams $D_{2 n}$ are realized as paragroups (in unique way) and the diagrams $D_{2 n+1}$ are impossible. This gave the first complete proof of this statement in the literature. Impossibility of $D_{2 n+1}$ has been independently proved by [43] and [76] based on fusion rules, the original proof of realization of $D_{2 n}$ of Ocneanu is now known [53, appendix], and Izumi's method for $E_{8}$ also gives a simpler proof of realization of $D_{2 n}$, but this orbifold method still gives the most natural conceptual understanding of the phenomena as seen below.

In [32], we first computed the higher relative commutants of the Hecke algebra subfactors of type $A$ of Wenzl [80] based on solutions to the YangBaxter equation by Jimbo-Miwa-Okado [46] as explained in section 1, and then applied the orbifold construction to these series. The sequence of subfactors of Wenzl with indices converging to $N^{2}$ corresponds to $S U(N)$. We proved in [32] that if $N=3$, the orbifold construction always gives a flat connection. Thus $S U(2)$ and $S U(3)$ behave quite differently in this question of flatness. We further noted with a little bit more work that the same result holds for odd prime $N$, and conjectured that the parity of $N$ causes this difference. F. $\mathrm{Xu}$ [83] proved that this is indeed the case. That is, if $N$ is odd, the orbifold construction always gives a flat connection, and if $N$ is even, the sequence of the resulting connections contains a flat connection and a nonflat connection alternately. Xu's method is more general and applicable to any subfactor arising from RCFT corresponding to $G_{k}$ Wess-Zumino-Witten models as explained as above, if we work at the symmetry of the paragroup arising from the center $Z$ of the Lie group $G$. 
By [32] and [83], the problem of flatness is reduced to verifying the identity

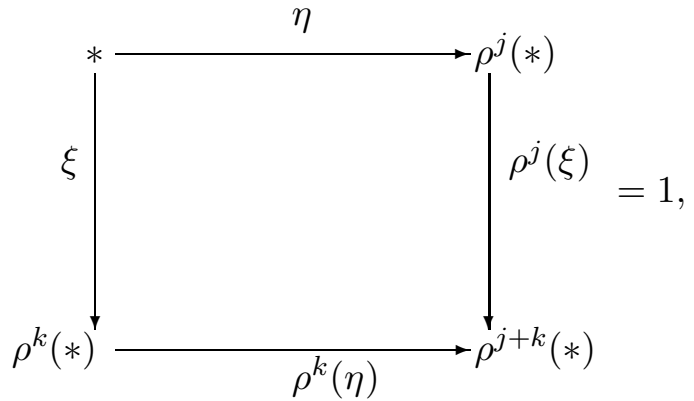

where $\rho$ denote the automorphism of the graph arising from the symmetry. $\mathrm{Xu}$ further noticed that it is enough to assume $k=-j$. By the same argument as in [11], this computation is reduced to that of the expectation value of the following knotted graph.

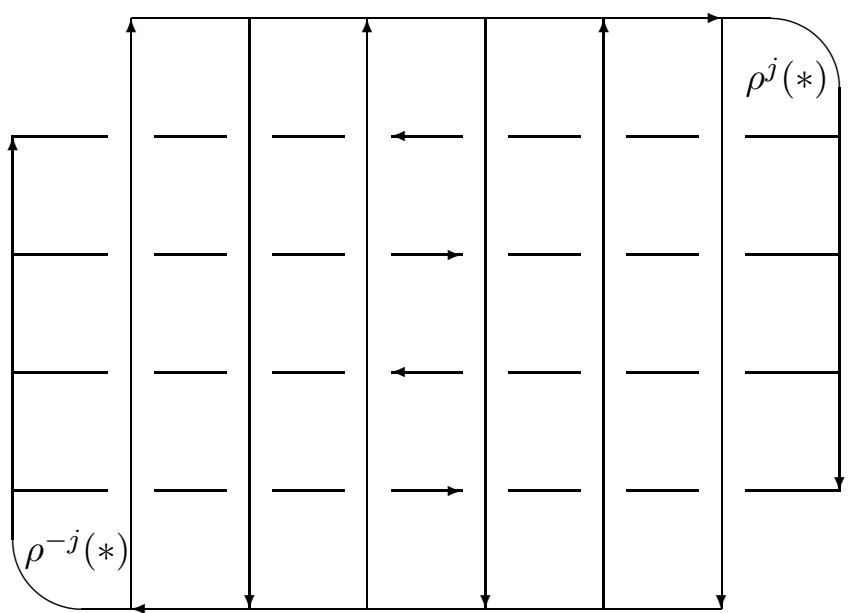

We again want to simplify the picture, but this expectation value is invariant only under regular isotopy, and this time we have a writhe for a curve at the upper right corner of the original graph. If we remove this writhe with the first Reidemeister move, we have a coefficient $\exp \left( \pm 2 \pi i h_{j}\right)$, where $h_{j}$ is the conformal dimension of $\rho^{j}(*)$, as in [82, page 639]. Thus we get flatness if and only if $h_{j}$ 's are integers. The conformal dimensions for $S U(N)$ have been known and we can decide when it is an integer.

Thus the difference between the Dynkin diagrams $D_{2 n}$ and $D_{2 n+1}$, first observed by Ocneanu in paragroup settings, is naturally understood as a 
very special case of general phenomena caused by conformal dimensions and the orbifold construction.

$\mathrm{Xu}$ further noticed that the condition on the Lie group $G$ and level $k$ equivalent to flatness of the resulting connections in the orbifold construction is the same as the one equivalent to existence of Chern-Simons gauge theory based on $G / Z$ at level k.

We conclude this section by an important remark. Although the orbifold construction has its origin in conformal field theory [26] and works well in paragroup setting as seen above, it does not produce the combinatorial data necessary for rational conformal field theory in the following sense. The simplest case is the series $D_{2 n}$ obtained as $Z_{2}$-orbifolds of the series $A_{4 n-3}$ which comes from rational conformal field theory corresponding to $S U(2)$. Although the Dynkin diagrams $D_{2 n}$ are realized as paragroups, we cannot have a corresponding rational conformal field theory as in [11] since we do not have symmetric $S$-matrices.

\section{Topological Quantum Field Theory (TQFT) and Subfactors}

Turaev-Viro [78] introduced a topological invariant of 3-dimensional closed manifolds based on triangulations using the quantum $6 j$-symbols of KirillovReshetikhin [56]. They define the invariant as a state sum for a triangulation; topological invariance of this state sum is based on certain algebraic relations of the $6 j$-symbols. To achieve this, they proved that classical Alexander moves [1] can be replaced simpler local moves of tetrahedra. (For a simple proof of this claim in 3 and 4 dimensions, see [39].) A. Ocneanu [67] claimed without a full proof that this type of invariants can be generalized to a subfactor setting and that algebraic axioms necessary for topological invariance are equivalent to the paragroup axioms. Although he has not published the exact statement of the theorem or a complete proof, a complete proof was given in [33] and the full machinery was worked out there. We discuss this aspect of paragroup theory here.

The basic idea of the construction in [78] is as follows. We have a set of finitely many colors with multiplication rules and each color has a certain weight. For a fixed triangulation of a given 3-dimensional compact manifold, we consider all the admissible configurations of colors assigned to edges appearing in the triangulation. (Admissibility is determined by the multiplication rule.) Each tetrahedron with six colors on six edges has a complex value called a $6 j$-symbol. For a given configuration, we take the product of all the $6 j$-symbol values, and then take a weighted sum of all the products over all configurations, which is called the state sum. In order to show that this complex number is really a topological invariant, we have to prove that the $6 j$-symbol has a certain symmetry and that the state sum does not depend on a choice of triangulation. Turaev-Viro proved that 
these two properties follow from three axioms on $6 j$-symbols and presented the Kirillov-Reshetikhin quantum $6 j$-symbol for $U_{q}\left(s l_{2}\right)$ [56] satisfied these axioms. The symmetry of $6 j$-symbols we need means that the value given to a tetrahedron does not depend on the way we project a tetrahedron on paper, and is called tetrahedral symmetry. The other two axioms in [78] are unitarity and the pentagon relation. In order to prove that this construction gives a complex number independent on triangulation, we have to compare two triangulations of the same manifold. Alexander [1] already showed that such two triangulations can be transformed to each other by finite steps of local moves, called Alexander moves. These moves, however, are infinitely many, and are not easy to handle, so Turaev-Viro replaced the Alexander moves in 3-dimensions by three local moves. The unitarity and the pentagon relation are axioms equivalent to invariance of the state sum under these three moves. Dijkgraaf-Witten proposed a similar invariant arising from 3-cocycles of finite groups based on physical ideas. A rigorous presentation of this invariant based on Alexander's theorem is given by [79].

To generalize this machinery to a general subfactor setting, we first need an appropriate $6 j$-symbol. This can be done as the generalization of a connection as in [67]. In paragroup theory, we look at four bimodules, and composition of four intertwiners among the tensor products of these four bimodules and ${ }_{N} L^{2}(M)_{M}$ and ${ }_{M} L^{2}(M)_{N}$. Extending this setting, we look at six bimodules and composition of four intertwiners among their tensor products. We also have a fusion algebra consisting of four kind of bimodules: $N-N, N-M, M-N$, and $M-M$. Each bimodule has a weight given by the corresponding Jones index. In this way, we have a generalized $6 j$-symbols with tetrahedral symmetry, unitarity, and flatness. The tetrahedral symmetry (after a certain normalization) comes from the Frobenius reciprocity of bimodules and intertwiners $[67,84]$ and unitarity is just as usual. So all we have to prove is the pentagon relation.

First write down the following identity as $3 \times 3$-flatness. Then split the square into two pieces as follows.
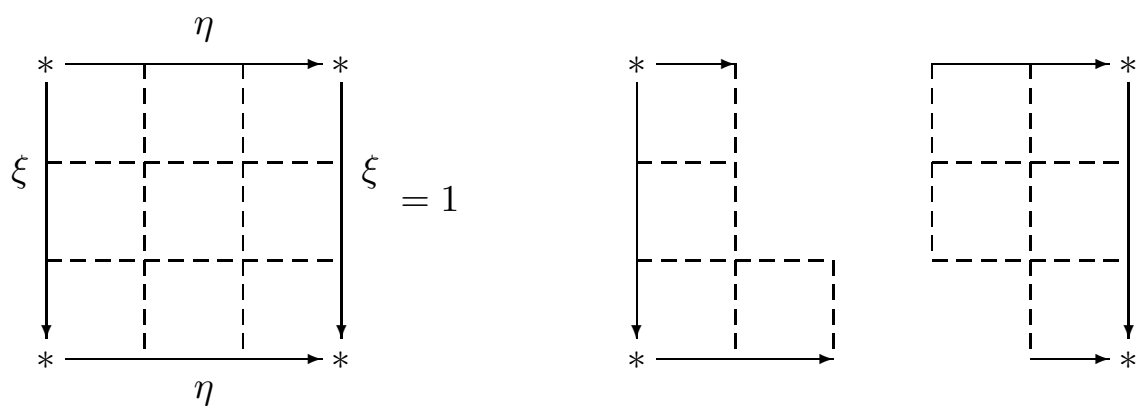
It is not difficult to see that the Cauchy-Schwarz inequality implies that these two pieces have the same partition function value. (This kind of use of the Cauchy-Schwarz inequality was first made in $[32, \S 5]$.) It is easy to see that all the four corner square of the picture have value 1 up to some normalization constant. Thus we have non-trivial values for two squares for the left picture and three square for the right picture. The pentagon relation is obtained immediately by writing down this identity among five squares.

Now we turn to the problem of the construction in the converse direction, constructing a subfactor from a topological invariant. Of course, we assume that the topological invariant is of Turaev-Viro type in the sense that it is a state sum based on triangulation. We also need a finitely generated fusion algebra with positive weights on the generators. Such conditions are often called rationality and unitarity respectively. By reversing the above argument, we get a biunitary connection and its $3 \times 3$-flatness in the sense that partition functions of size $3 \times 3$ have value 1 . All we need is (ordinary) flatness now. This can be done as follows. First we split the $3 \times 3$-picture in the following way.

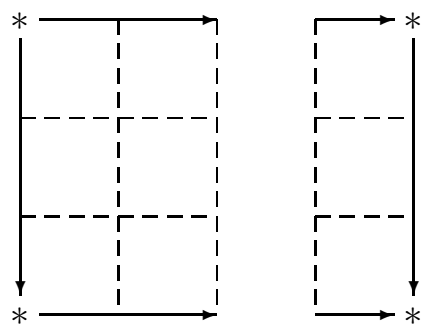

Then again by the Cauchy-Schwarz argument, we can reduce the size $3 \times n$ of a large diagram to $3 \times 3$ as follows. 

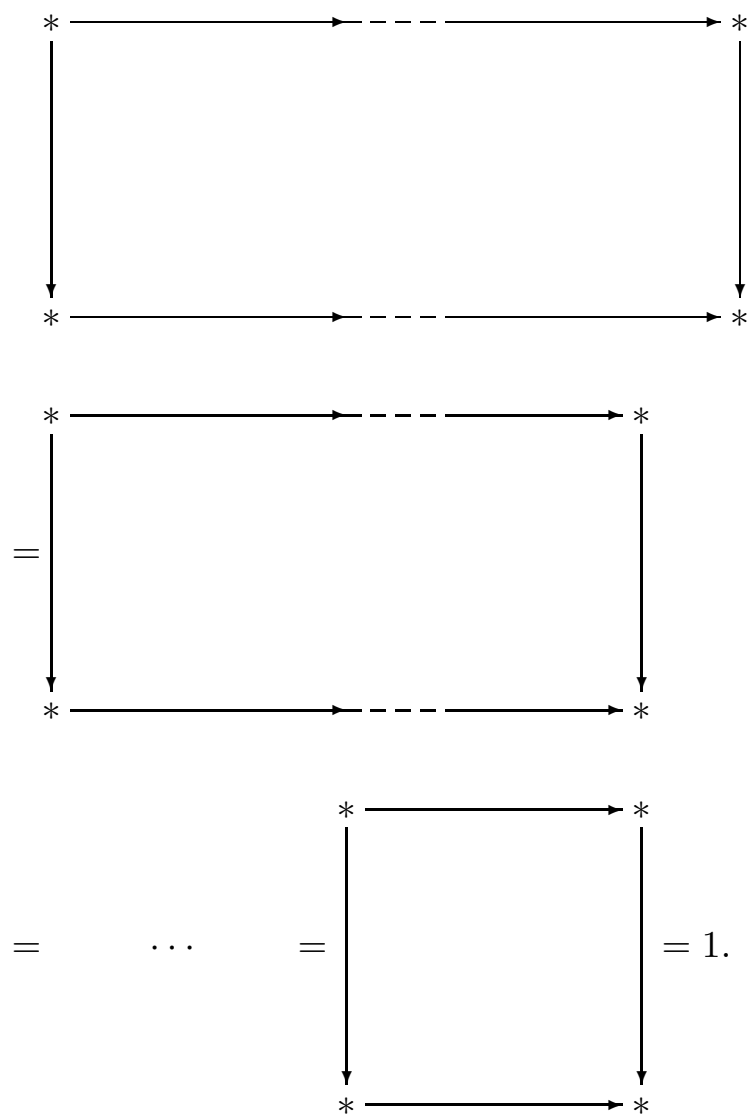

Then by the same kind of argument, we can reduce the size $m \times n$ of a large diagram to $3 \times n$. In this way, we can prove ordinary flatness from $3 \times 3$-flatness. This is an analogue of the argument of de Boer-Goeree [11] mentioned in Section 4 in the sense that we get a global identity from local identities.

In this way, we can say that flatness is equivalent to the pentagon relation which means invariance of the state sum under the following move which we call move I. 

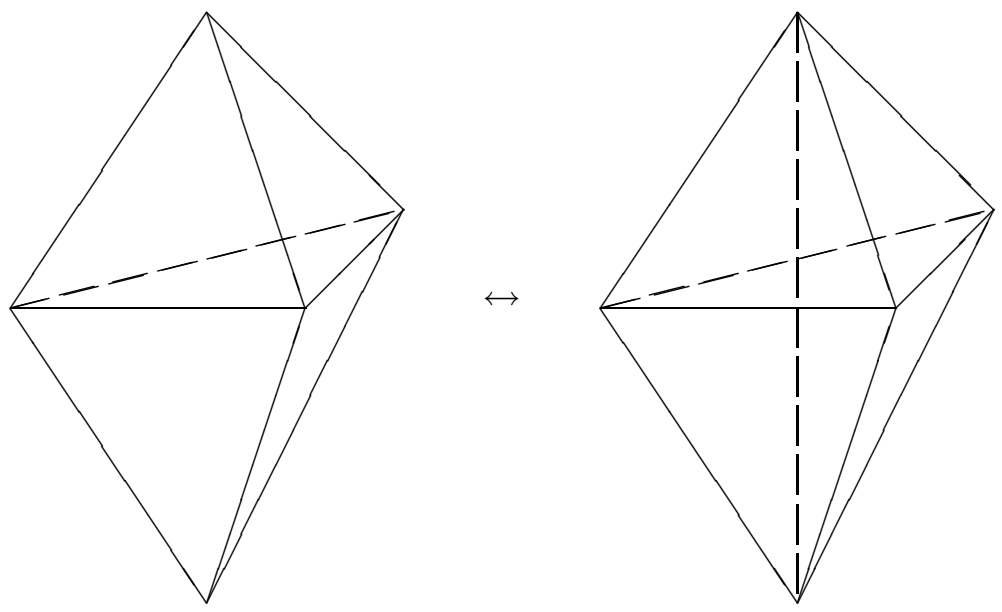

where the left picture has two tetrahedra and the right has three tetrahedra.

At the end of this section, we mention two subtle differences between the Turaev-Viro construction and topological invariants arising from subfactors. The first difference is unitarity, or positivity of the trace. If we start from a subfactor, each element of the fusion algebra has a positive weight given by the Jones index, and we need this positivity for the construction in the converse direction. Turaev-Viro machinery, however, does not require this positivity, and actually, the Kirillov-Reshetikhin quantum $6 j$-symbol does not satisfy this positivity in general. At the end of [78], they compute the invariant for the case Dynkin diagram $A_{2}$ for two choices of roots of unity and get different values. One of the two choices does not satisfy positivity condition and does not come from a subfactor. In general, we have to choose a root of unity $q$ to be $\exp (\pi i / k)$ to get this positivity. The other difference comes from $\mathbf{Z}_{2}$-grading of vertices of the principal graph. In the above state sum approach, each vertex has an assignment of $M$ or $N$ in our setting unlike in [78]. We do not have to take a sum over configurations of $M$ or $N$ on vertices since each fixed configuration on vertices gives the same value as proved in [33]. (The key argument for this is as follows. In one of the three local moves, one vertex disappears. The assignment of $M$ or $N$ to this vertex does not change the value we obtain.) In particular, we can use only $N-N$ bimodules or $M-M$ bimodules. In the Turaev-Viro case of $A_{n}$ Dynkin diagrams, this means that we use only a fusion subalgebra generated by "colors" labeled by integers instead of the entire algebra generated by "colors" labeled by half-integers. This causes another difference between the Turaev-Viro invariant and the invariant coming from the Jones subfactor of type $A_{n}$. 


\section{Automorphisms of Subfactors}

As seen above, the orbifold construction gives new series of interesting subfactors as simultaneous fixed point algebras of finite group actions on the original subfactors. This suggests that automorphisms appearing in this construction have interesting operator algebraic properties. The aim of this section is to show that this is indeed the case. Beginning with a series of papers by A. Connes, there has been extensive work on the automorphisms of operator algebras, and many results on the classification of automorphisms and group actions on injective factors have been obtained. In this work, the roles of two classes of automorphisms have been emphasized. One is a class of approximately inner automorphisms and the other is that of centrally trivial automorphisms. In the subfactor setting, Loi introduced analogues of these two classes for subfactors and we use the same names "approximately inner" and "centrally trivial" for these analogues. Let $N \subset M$ be a subfactor of type $\mathrm{II}_{1}$. An automorphism $\alpha \in \operatorname{Aut}(M, N)$ is said to be approximately inner if it is of the form $\lim _{n} \operatorname{Ad}\left(u_{n}\right)$ with $u_{n} \in N$ and it is said to be centrally trivial if $\alpha$ acts trivially on $N^{\omega} \cap M^{\prime}$, where $\omega$ is a free ultrafilter over $\mathbf{N}$.

In the following, let $N \subset M$ be a strongly amenable subfactor of type $\mathrm{II}_{1}$ in the sense of S. Popa [71]. This condition is equivalent to the generating property in the sense that the higher relative commutants reproduce the subfactor for some choice of tunnel [71]. P. Loi studied the classification problem of $\alpha \in \operatorname{Aut}(M, N)$ to classify type $\mathrm{III}_{\lambda}$ subfactors, $0<\lambda<1$, via subfactor version of Connes' discrete decomposition in [61], and introduced his invariant of automorphisms of subfactors. This invariant is given by the action of (the extension of) an automorphism on the higher relative commutants, and he proved that triviality of this invariant is equivalent to approximate innerness of the automorphism. He also proved that if any nontrivial power of $\alpha \in \operatorname{Aut}(M, N)$ is outer on $M$ and $N \subset M$ has finite depth, then no non-trivial power of this $\alpha$ is centrally trivial. He further noticed that if $\alpha \in \operatorname{Aut}(M, N)$ is approximately inner and no non-trivial power of $\alpha$ is centrally trivial, a Connes type argument as in [17] uniquely determines $\alpha$ up to outer conjugacy.

Consider our orbifold action $\alpha$ of $\mathbf{Z}_{2}$ on subfactors of type $A_{4 n-3}$. Loi's invariant is always trivial on subfactors of type $A_{n}$, so all automorphisms of these subfactors are approximately inner. Because the simultaneous fixed point algebra gives a different subfactor $D_{2 n}$, this action $\alpha$ is different from the "standard" action of $\mathbf{Z}_{2}$, which is of the form id $\otimes \sigma$ on $N \bar{\otimes} R \subset M \bar{\otimes}$, where $\sigma$ is a (unique) outer action of $\mathbf{Z}_{2}$ on the common hyperfinite $\mathrm{II}_{1}$ factor $R$ and $N \bar{\otimes} R \subset M \bar{\otimes} R$ is conjugate to $N \subset M$. By applying Loi's Connes type argument to $\mathbf{Z}_{2}$, we conclude that this action $\alpha$ is centrally trivial.

This method, however, is quite indirect, and does not work in the case of $A_{4 n-1}$, where the simultaneous fixed point algebras are of the same type. 
A more direct method using partition functions gives a better proof. Let $\alpha$ be an automorphism appearing in the orbifold construction for subfactors arising from RCFT as in section 4 . Then computations based on topological moves of knotted graphs show the following identities for any $k$.
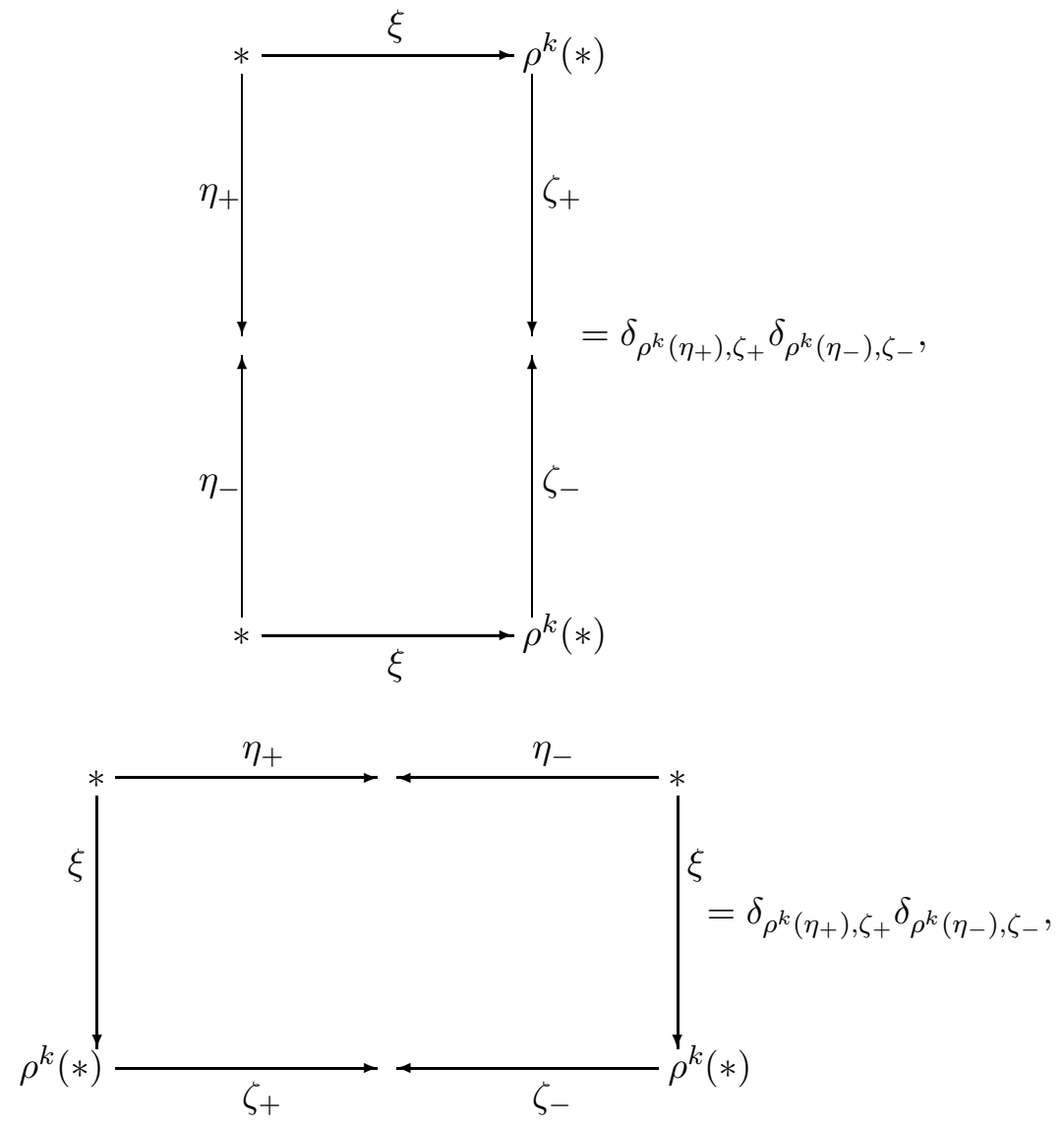

where $\rho$ is the induced symmetry of the graph.

We can show that the first identity implies that $\alpha$ acts trivially on higher relative commutants, and the second implies that $\alpha$ acts trivially on central sequences. (See [32, Remark 5.9], [54, Lemma 4.3] respectively.) In this sense, approximate innerness and central triviality appear in a symmetric way, and we get that $\alpha \in \operatorname{Ct}(M, N) \cap \overline{\operatorname{Int}}(M, N)$. Note that we get this regardless of whether the orbifold construction produces a flat connection.

In a single factor case, Connes [16] introduced an invariant $\chi(M)=$ $(\operatorname{Ct}(M) \cap \overline{\operatorname{Int}}(M)) / \operatorname{Int}(M)$ and proved several interesting results. (Also see [47].) The above observation means that the orbifold construction produces 
non-trivial elements in the subfactor version of $\chi(M)$. We define $\chi(M, N)=$ $(\mathrm{Ct}(M, N) \cap \overline{\operatorname{Int}}(M, N)) / \operatorname{Int}(M, N)$, where $\mathrm{Ct}(M, N), \overline{\operatorname{Int}}(M, N), \operatorname{Int}(M, N)$ mean the class of centrally trivial automorphisms, the approximately inner automorphisms, and inner automorphisms given by unitaries in $N$, respectively. This is an abelian group as in the single factor case, and we have an analogue [54, Theorem 1.1] of the exact sequence in [16].

Next we aim to show that the automorphisms appearing in the orbifold construction for subfactors arising from RCFT as in section 4 actually give the entire $\chi(M, N)$. For example, in the case of Hecke algebra subfactors of type $A$ by Wenzl [80] with indices $\sin ^{2}(N \pi / k) / \sin ^{2}(\pi / k)$, we get $\chi(M, N)=$ $\mathbf{Z}_{d}$ with $d=(k, N)$. (In [54, Proposition 4.5], this was proved only for the case where $N$ is prime, but the same method based on the above computation of partition functions gives this.) This claim is proved by getting an upper bound for the size of the group $\operatorname{Ct}(M, N)$ as follows.

Popa and Choda-Kosaki independently introduced the same notion of outerness of an automorphism in different situations. Popa calls an $\alpha \in$ $\operatorname{Aut}(M, N)$ properly outer if the identity $a x=\alpha(x) a$ for some $a \in M_{k}$ and all $x$ implies $a=0$. Choda-Kosaki calls the same condition strongly outer. Popa [72] proved that if a subfactor is strongly amenable, then not being properly outer is equivalent to central triviality. Choda-Kosaki $[15,57]$ proved that $\alpha \in \operatorname{Aut}(M, N)$ is not strongly outer if and only if $\alpha$ appears at even vertices of the dual principal graph in sector/bimodule approach. (If a subfactor is of type III, this condition means that $\alpha$ appears in irreducible decompositions of powers of Longo's canonical endomorphism.) Combining these two results, we know that the order of the $\operatorname{group} \operatorname{Ct}(M, N) / \operatorname{Int}(M, N)$ is bounded by the number of even vertices of the dual principal graph with normalized Perron-Frobenius weights 1 . In the case of subfactors arising from RCFT as in Section 4, this upper bound is attained and we get $\chi(M, N)$.

This upper bound was proved in the finite depth case first in [54] based on Ocneanu's approach with central sequence subfactors and asymptotic inclusion $[64,66]$. (The statements, made without proof, by Ocneanu needed in this approach were proved in [54].) Although this approach is more complicated than combining results of Choda-Kosaki and Popa, it is expected to give more information on subfactors with finite depth. For example, Ocneanu has suggested that considerable information on TQFT of Section 5 can be obtained from central sequence subfactors and asymptotic inclusions.

Note that central triviality is easier to handle with partition functions than the property of not being properly outer is, although these two are equivalent in our situation. For example, in the orbifold construction for $A_{2 n-3}$, it is easy to write down a non-zero $a \in M_{k}$ with $a x=\alpha(x) a, x \in N$, explicitly as in [52] if $n$ is even, but it is not clear at all how to get such an $a$, if $n$ is odd, while central triviality is easily established in both cases. With this advantage, we can prove the following: If a subfactor arises from 
RCFT as in Section 4, automorphisms appearing on even vertices of the dual principal graph are the same as those appearing in the orbifold construction. That is, the orbifold construction gives a very concrete realization of these automorphisms appearing in a more abstract way in the sector/bimodule approach.

Note that a recent construction of a subfactor with finite depth and index $(5+\sqrt{13}) / 2$ by Haagerup [40] shows that the above upper bound is not always attained. (This is related to a problem whether one can adjust a certain automorphism $M$ so that it fixes $N$ globally. See [57].)

Thus we have many examples of subfactors with non-trivial $\chi(M, N)$, and it is not difficult to realize all finite abelian groups as $\chi(M, N)$ for subfactors with finite depth using tensor products. In the case of single factor, Connes $[16,47]$ constructed several examples with non-trivial $\chi(M)$, but these are rather unusual $\mathrm{II}_{1}$ factors in the sense that they are non-AFD with property $\Gamma$. Subfactors arising from RCFT such as Hecke algebras subfactors of Wenzl are very natural and basic objects in subfactor theory, and they should not regarded as analogues of such exotic single factors. The right analogues of these subfactors in the single factor case turn out to be (injective) type III factors.

Loi's invariant has considerable similarity with the Connes-Takesaki module [20], and paragroups can be regarded as discrete analogues of flows of weights [20], as pointed out in [51]. For example, an automorphism of a strongly amenable subfactors is approximately inner if and only if its Loi invariant is trivial [61, Theorem 5.4], while an automorphism of an injective type III factor is approximately inner if and only if its module is trivial, as announced by Connes [18, section 3.8] and proved by [55, Theorem 1].

As to the other important class of automorphisms, centrally trivial automorphisms, Connes [18] announced that an automorphism $\alpha$ of an injective type III factor is centrally trivial if and only if it is an extended modular automorphism up to inner perturbation, and this was proved in [55, Theorem 1]. From this viewpoint, we come to the idea that an orbifold action arising from RCFT is a (discrete) analogue of modular automorphisms. With this understanding, we can interpret the result of Choda-Kosaki [15, 57] naturally as an analogue of a result of Haagerup-Størmer [41, Proposition 5.4].

It is easy to see that the orbifold $\mathbf{Z}_{2}$ actions on $A_{4 n-3}$ and the $\mathbf{Z}_{2}$ actions flipping the two tails of $D_{2 n}$ are in Takesaki type duality. This duality is again a discrete analogue of that between the Connes-Takesaki module [20] and the Sutherland-Takesaki modular invariant [77].

Popa [72] obtained the classification result of discrete amenable group actions on strongly amenable subfactors in the sense that properly outer such actions are classified by the Loi invariant. Because such actions are centrally free, this is again an analogue of the classification of centrally 
free actions of discrete amenable groups on injective type III factors by the Connes-Takesaki module.

Longo had already noticed in [62] that his canonical endomorphisms are similar to modular automorphism groups. From our viewpoints, automorphisms appearing in the powers of canonical endomorphisms have further similarity to modular automorphisms, and in this approach the analogy makes sense even in type $\mathrm{II}_{1}$ settings.

This kind of central sequence approach is useful for the classification of group actions on subfactors. For example, there are two and only two outer actions of $\mathbf{Z}_{2}$ on subfactors of type $A_{2 n+1}$, and one is given by the standard form and the other is given by the orbifold construction. There is also an analogue of modular obstruction. For more on this classification, see [54, §5].

A basic philosophy in the above type of analogy is that any phenomenon on type III (single) factors appears in type $\mathrm{II}_{1}$ subfactor in a discrete form. A mixture of the both will appear in type III subfactors. Winsløw's simultaneous generalization of the Connes-Takesaki module and Loi invariant in [81] is the first example of such a mixture.

It is a very important problem to distinguish non-amenable subfactors with the same indices using $\chi(M, N)$ as an analogue of Connes' construction in [16] for single $\mathrm{II}_{1}$ factors. Our exact sequence [54, Theorem 1.1] is expected to be useful for this purpose, but so far, no example has been computed in the non-amenable subfactor case.

Finally, we discuss the problem of finding non-commutative symmetries of paragroups. In Xu's construction [83], the finite group acting on subfactors arising from RCFT is given as the center of the Lie group with which we started. In particular, this finite group is always abelian. In general, there seems to be no reason why a symmetry group of a given paragroup is abelian and the problem to find an interesting non-abelian symmetry has caught attention of physicists. From the viewpoint of automorphisms, $\chi(M, N)$ is always abelian, but $\operatorname{Ct}(M, N) / \operatorname{Int}(M, N)$ is not necessary abelian. (A trivial example is a subfactor $R^{G} \subset R$ with $G$ finite, non-commutative, and acting on $R$ outerly.) We feel that "interesting" symmetries should give $\chi(M, N)$ and so are commutative by some deeper reason, but nothing is clear at present.

\section{References}

[1] J. W. Alexander, The combinatorial theory of complexes, Ann. Math. (2) 31 294-322, (1930).

[2] G. E. Andrews, R. J. Baxter, \& P. J. Forrester, Eight vertex SOS model and generalized Rogers-Ramanujan type identities, J. Stat. Phys. 35 193-266, (1984).

[3] H. Araki \& D. E. Evans, On a $C^{*}$-algebra approach to phase transition in the two dimensional Ising model, Comm. Math. Phys. 106 241-266, (1986).

[4] J. Bion-Nadal, An example of a subfactor of the hyperfinite $\mathrm{II}_{1}$ factor whose principal graph invariant is the Coxeter graph $E_{6}$, in "Current Topics in Operator Algebras", 
World Scientific Publishing, pp. 104-113, (1991).

[5] B. Blackadar, Symmetries of the CAR algebra, Ann. Math. 131 589-623, (1990).

[6] O. Bratteli, G. A. Elliott, D. E. Evans, \& A. Kishimoto, Non-commutative spheres, Inter. J. Math. 2 139-166, (1991).

[7] O. Bratteli, G. A. Elliott, D. E. Evans, \& A. Kishimoto, Finite group actions on AF algebras obtained by folding the interval, (to appear in $K$-theory).

[8] O. Bratteli, G. A. Elliott, D. E. Evans, \& A. Kishimoto, Non-commutative spheres II: rational rotations, (to appear in J. Operator Theory).

[9] O. Bratteli, D. E. Evans, \& A. Kishimoto, Crossed products of totally disconnected spaces by $\mathbf{Z}_{2} * \mathbf{Z}_{2}$, preprint, Swansea, (1991).

[10] O. Bratteli \& A. Kishimoto, Non-commutative spheres III: irrational rotations, preprint, Trondheim, (1991).

[11] J. de Boer \& J. Goeree, Markov traces and $\mathrm{II}_{1}$ factors in conformal field theory, Comm. Math. Phys. 139 267-304, (1991).

[12] A. Cappelli, C. Itzykson, \& J.-B. Zuber, The $A-D-E$ classification of minimal and $A_{1}^{(1)}$ conformal invariant theories, Comm. Math. Phys. 113 1-26, (1987).

[13] A. L. Carey \& D. E. Evans, The operator algebras of the two dimensional Ising model, in "Braids" eds. J. Birman, A. Libgober, Comtemp. Math. 78 117-165, (1988).

[14] M. Choda, Duality for finite bipartite graphs, to appear in Pac. J. Math.

[15] M. Choda \& H. Kosaki, Strongly outer actions for inclusion of factors, preprint, (1992).

[16] A. Connes, Sur la classification des facteurs de type II, C. R. Acad. Sc. Paris 281 13-15, (1975).

[17] A. Connes, Outer conjugacy classes of automorphisms of factors, Ann. Sci. École Norm. Sup. 8 383-419, (1975).

[18] A. Connes, On the classification of von Neumann algebras and their automorphisms, Symposia Math. XX 435-478, (1976).

[19] A. Connes \& D. E. Evans, Embeddings of $U(1)$-current algebras in non-commutative algebras of classical statistical mechanics, Comm. Math. Phys. 121 507-525, (1989).

[20] A. Connes \& M. Takesaki, The flow of weights on factors of type III, Tohoku Math. J. 29 473-555, (1977).

[21] E. Date, M. Jimbo, T. Miwa, \& M. Okado, Solvable lattice models, in "Theta functions - Bowdoin 1987, Part 1," Proc. Sympos. Pure Math. Vol. 49, Amer. Math. Soc., Providence, R.I., pp. 295-332.

[22] P. Di Francesco \& J.-B. Zuber, $S U(N)$ lattice integrable models associated with graphs, Nucl. Phys. B338 602-646, (1990).

[23] P. Di Francesco \& J.-B. Zuber, $S U(N)$ lattice integrable models and modular invariance, (to appear in Proc. Trieste Conf. Recent Developments in Conformal Field Theories, Trieste, 1989).

[24] R. Dijkgraaf \& E. Verlinde, Modular invariance and the fusion algebra, Proceedings of the Annecy Conference on Conformal Field Theory. Nucl. Phys. B(Proc. Suppl.) 5B 87-97, (1988).

[25] R. Dijkgraaf \& E. Witten, Topological gauge theories and group cohomology, Comm. Math. Phys. 129 393-429, (1990).

[26] L. Dixon, J. A. Harvey, C. Vafa, \& E. Witten, Strings on orbifolds, Nucl. Phys. B261, 678-686, (1985); B274 285-314, (1986).

[27] B. Durhuus, H. P. Jakobsen, \& R. Nest, Topological quantum field theories from generalized 6j-symbols, preprint, (1991).

[28] D. E. Evans, The $C^{*}$-algebras of topological Markov chains, Tokyo Metropolitan University Lecture Notes, (1984).

[29] D. E. Evans, Quasi-product states on $C^{*}$-algebras, in "Operator algebras and their connections with topology and ergodic theory", Springer Lecture Notes in Math., 1132 129-151, (1985).

[30] D. E. Evans, $C^{*}$-algebraic methods in statistical mechanics and field theory, Inter. J. Mod. Phys. 4 1069-1118, (1990). 
[31] D. E. Evans \& J. D. Gould, Embeddings and dimension groups of non-commutative AF algebras associated to models in classical statistical mechanics, preprint, Swansea.

[32] D. E. Evans \& Y. Kawahigashi, Orbifold subfactors from Hecke algebras, preprint, (1992).

[33] D. E. Evans \& Y. Kawahigashi, From subfactors to 3-dimensional topological quantum field theories and back, preprint, (1992).

[34] D. E. Evans \& Y. Kawahigashi, The $E_{7}$ commuting squares produce $D_{10}$ as principal graph, preprint, (1992).

[35] D. E. Evans \& J. T. Lewis, On a $\mathrm{C}^{*}$-algebra approach to phase transition in the two-dimensional Ising model II, Comm. Math. Phys. 102 521-535, (1986).

[36] P. Fendley, New exactly solvable orbifold models, J. Phys. A22 4633-4642, (1989).

[37] P. Fendley \& P. Ginsparg, Non-critical orbifolds, Nucl. Phys. B324 549-580, (1989).

[38] F. Goodman, P. de la Harpe, \& V. F. R. Jones, "Coxeter graphs and towers of algebras", MSRI publications 14, Springer, (1989).

[39] M. Gross \& S. Varsted, Elementary moves and ergodicity in D-dimensional simplicial quantum gravity, Nucl. Phys. B378 367-380, (1992).

[40] U. Haagerup, in preparation.

[41] U. Haagerup \& E. Størmer, Pointwise inner automorphisms of von Neumann algebras (with an appendix by C. Sutherland), J. Funct. Anal. 92 177-201, (1990).

[42] T. Hayashi, Quantum group symmetry of partition functions of IRF models and their application to Jones' index theory, preprint, (1992).

[43] M. Izumi, Application of fusion rules to classification of subfactors, Publ. RIMS Kyoto Univ. 27 953-994, (1991).

[44] M. Izumi, On flatness of the Coxeter graph $E_{8}$, to appear in Pac. J. Math.

[45] M. Izumi \& Y. Kawahigashi, Classification of subfactors with the principal graph $D_{n}^{(1)}$, to appear in J. Funct. Anal.

[46] M. Jimbo, T, Miwa, \& M. Okado, Solvable lattice models whose states are dominant integral weights of $A_{n-1}^{(1)}$, Lett. Math. Phys. 14 123-131, (1987).

[47] V. F. R. Jones. Notes on Connes' invariant $\chi(M)$, unpublished.

[48] V. F. R. Jones, Index for subfactors, Invent. Math. 72 1-15, (1983).

[49] V. F. R. Jones, A polynomial invariant for knots via von Neumann algebras Bull. Amer. Math. Soc. 12 103-112, (1985).

[50] A. Kato, Classification of modular invariant partition functions in two dimensions, Mod. Phys. Lett. A 2 585-600, (1987).

[51] Y. Kawahigashi, Automorphisms commuting with a conditional expectation onto a subfactor with finite index, to appear in J. Operator Theory.

[52] Y. Kawahigashi, On flatness of Ocneanu's connections on the Dynkin diagrams and classification of subfactors, University of Tokyo, preprint, (1990).

[53] Y. Kawahigashi, Exactly solvable orbifold models and subfactors, to appear in Proceedings of the Conference on Functional Analysis and Related Topics, Kyoto, 1991.

[54] Y. Kawahigashi, Centrally trivial automorphisms and an analogue of Connes' $\chi(M)$ for subfactors, to appear in Duke Math. J.

[55] Y. Kawahigashi, C. E. Sutherland, \& M. Takesaki, The structure of the automorphism group of an injective factor and the cocycle conjugacy of discrete abelian group actions, Acta Math. 169 105-130, (1992).

[56] A. N. Kirillov \& N. Yu. Reshetikhin, Representations of the algebra $U_{q}\left(s l_{2}\right), q-$ orthogonal polynomials and invariants for links, in "Infinite dimensional Lie algebras and groups" (V. G. Kac, ed.), Adv. Ser. in Math. Phys., vol. 7, pp. 285-339, (1988).

[57] H. Kosaki, Automorphisms in irreducible decompositions of sectors, in this volume.

[58] R. Kuik, On the $q$-state Potts model by means of non-commutative algebras, Thesis Groningen, (1986)

[59] A. Kumjian, An involutive automorphism of the Bunce-Deddens algebra, CR Math. Rep. Acad. Sci. Canada 10 217-218, (1988).

[60] J. T. Lewis, P. N. M. Sisson, A $C^{*}$-algebra of the 2-dimensional Ising model, Comm. Math. Phys. 44 279-292, (1975). 
[61] P. H. Loi, On automorphisms of subfactors, preprint, (1990).

[62] R. Longo, Simple injective subfactors, Adv. Math. 63 152-171, (1987).

[63] G. Moore \& N. Seiberg, Classical and quantum conformal field theory, Comm. Math. Phys. 123 177-254, (1989).

[64] A. Ocneanu, Quantized group, string algebras and Galois theory for algebras, in "Operator algebras and applications, Vol. 2 (Warwick, 1987)," London Math. Soc. Lect. Note Series Vol. 136, Cambridge University Press, pp. 119-172, (1988).

[65] A. Ocneanu, "Graph geometry, quantized groups and nonamenable subfactors", Lake Tahoe Lectures, June-July, (1989).

[66] A. Ocneanu, "Quantum symmetry, differential geometry of finite graphs and classification of subfactors", University of Tokyo Seminary Notes 45, (Notes recorded by Y. Kawahigashi), (1991).

[67] A. Ocneanu, An invariant coupling between 3-manifolds and subfactors, with connections to topological and conformal quantum field theory, unpublished announcement, (1991).

[68] S. Okamoto, Invariants for subfactors arising from Coxeter graphs, in "Current Topics in Operator Algebras," World Scientific Publishing, pp. 84-103, (1991).

[69] V. Pasquier, Operator content of the ADE lattice models, J. Phys. A. Math. Gen. 20 5701-5717, (1987).

[70] S. Pirogov, States associated with the two dimensional Ising model, Theor. Math. Phys. 11(3) 614-617, (1972).

[71] S. Popa, Classification of amenable subfactors of type II, IHES preprint, (1992).

[72] S. Popa, Classification of actions of discrete amenable groups on amenable subfactors of of type II, IHES preprint, (1992).

[73] $\mathrm{Ph}$. Roche, Ocneanu cell calculus and integrable lattice models, Comm. Math. Phys. 127 395-424, (1990).

[74] J. Schou, Commuting squares and index for subfactors, Ph. D. Thesis, Odense University, 1990.

[75] V. S. Sunder, A model for AF-algebras and a representation of the Jones projections, J. Operator Theory 18 289-301, (1987).

[76] V. S. Sunder \& A. K. Vijayarajan, On the non-occurrence of the Coxeter graphs $\beta_{2 n+1}, E_{7}, D_{2 n+1}$ as principal graphs of an inclusion of $\mathrm{II}_{1}$ factors, (to appear in Pac. J. Math.).

[77] C. E. Sutherland \& M. Takesaki, Actions of discrete amenable groups on injective factors of type $\mathrm{III}_{\lambda}, \lambda \neq 1$, Pac. J. Math. 137 405-444, (1989).

[78] V. G. Turaev \& O. Y. Viro, State sum invariants of 3-manifolds and quantum $6 j$ symbols, preprint.

[79] M. Wakui, On Dijkgraaf-Witten invariant for 3-manifolds, (to appear in Osaka J. Math.).

[80] H. Wenzl, Hecke algebras of type $A_{n}$ and subfactors, Invent. Math. 92 345-383, (1988).

[81] C. Winsløw, Approximately inner automorphisms on inclusions of type $\mathrm{III}_{\lambda}$ factors, preprint, (1992).

[82] E. Witten, Gauge theories and integrable lattice models, Nucl. Phys. B322 629-697, (1989).

[83] F. Xu, Orbifold construction in subfactors, preprint, (1992).

[84] S. Yamagami, A note on Ocneanu's approach to Jones' index theory, preprint, (1992). 